\title{
uince Mil Palabras de Viaje sin Destino. La Terminología Arqueológica dentro del Tesauro de Patrimonio Histórico de Andalucía
}

\author{
L. García Sanjuán \\ Department of \\ Archaeological Sciences \\ University of Bradford \\ V. Hurtado Pérez \\ Departamento de \\ Prehistoria y Arqueología \\ Universidad de Sevilla
}

\section{Resumen}

En este artículo se describe el proceso de integración de la terminología arqueológica dentro del Tesauro de Patrimonio Histórico de Andalucía. Dada la aproximación temática, y no disciplinar, seguida para la elaboración de este documento, dicho proceso ha requerido una meticulosa observación de las principales areas de solapamiento léxico entre la Arqueología y algunas de sus disciplinas académicas más afines, especialmente la Etnología o la Arquitectura. Este proceso ha implicado el diseño de una jerarquía conceptual relativamente ajena a algunas de las tradiciones disciplinares representadas, pero que en conjunto es capaz de acomodar la terminología arqueológica sin excesivas dificultades. Se discuten algunos de los problemas más sobresalientes experimentados, incluyendo el número de términos disponible (limitación en la profundidad y precisión de la terminología), la existencia de polisemias (matización del significado de la terminología) o la presencia de ambiguedades en la definición de los términos.

Palabras clave:

Andalucía / Arqueología / Arquitectura / Etnología / Integración disciplinar / Terminología / Tesauros / Patrimonio histórico

\section{Introducción}

La elaboración de la versión 0 del Tesauro del Patrimonio Histórico Andaluz (TPHA) dió comienzo en Julio de 1995 con la conformación del Grupo de Trabajo que habría de acometer su redacción. Dentro de este Grupo de Trabajo de 1 I miembros, la arqueología como disciplina estaba representada por tres arqueológos, dos de perfil académico (los autores de este artículo) y un conservador de museos (M. Ramos Lizana). La especialidad arqueológica quedaba por tanto suficientemente representada en la configuración del equipo humano designado para esta tarea. Incluso, podría hasta cierto punto argumentarse que la Arqueología como disciplina estaba ligeramente sobrerrepresentada, lo que quizás en cierto sentido reflejara (desde luego de forma no intencionada) el fuerte peso específico que el Patrimonio Arqueológico en particular ha tenido y mantiene en el marco general de la gestión del Patrimonio Histórico en nuestra comunidad autónoma desde que la administración autonómica asumiera plenas competencias en la materia.

Entre Julio de 1995 y Enero de 1997 se elaboró la estructura jerárquica y se compiló el vocabulario básico, que finalmente excedió de forma muy considerable las previsiones iniciales (c. 15.000 términos frente a los 6.000-7.000 planeados como objetivo en un principio). La lentitud con la que esta parte del trabajo progresó (a pesar de la intensidad y constancia de las reuniones, afortunadamente sostenida con férrea manu militari por el coordinador del Grupo) fue debida, además de a la intrínseca dificultad de la tarea, a las importantes diferencias de configuración y desarrollo interno de todo orden (epistemológico, teórico, metodológico) que se dan entre las varias disciplinas implicadas. La menor de esas diferencias no resultó, ciertamente (y para doloroso descubrimiento de algunos de nosotros), el uso del lenguaje.

En Enero de 1997, la Comisión de Seguimiento, formada por expertos de nivel senior de varias institutiones de ámbito nacional, entre ellos arqueólogos/as de la Universidad de Granada y de la DGBBCC de la Junta de Andalucía - cf. descripción más detallada en la propia Introducción del TPHA (IAPH, 1998:15-16) - dió luz verde (después de hacer una serie de interesantes sugerencias y recomendaciones), a la estructura jerárquica y al vocabulario compilado, por lo que se dió inicio a la fase de establecimiento de relaciones entre términos, que se prolongaría hasta Octubre de 1997, resultando en un total de 13.000 relaciones.

A comienzos de 1998 el TPHA comienza a experimentar su primer gran test práctico, con su introducción en las bases de datos del IAPH, precisamente la función principal para la cual había sido concebido. Es, no obstante, tras su publicación en Noviembre 
de 1998, cuando el TPHA alcanza a toda la comunidad de colegas arqueólogos y arqueólogas. En Abril de 1999 se produce una reunión del Grupo de Trabajo para efectuar el primer seguimiento del funcionamiento del TPHA, valorando y estudiando los primeros problemas surgidos tras su aplicación a las bases de datos del Centro de Documentación del $\mathrm{APH}$. Varios tipos de errores son detectados, proponiéndose soluciones (algunos de estos son comentados en la sección final de este artículo).

\section{Arqueología, Gestión y Tesauros}

Para intentar comprender su virtualidad dentro del ámbito de la gestión arqueológica, el TPHA debe ser considerado desde la perspectiva general de la tendencia internacional hacia el desarrollo de tesauros que se viene dando en Arqueología desde mediados de los años 1980. Esta tendencia obedece fundamentalmente a la necesidad de alcanzar una estandarización de las estructuras y las terminologías de descripción de datos, consecuencia a su vez del creciente volumen y diversidad de la información almacenada (como resultado de la gestión arqueológica - típicamente arqueología de urgencia), así como de la introducción de las aplicaciones informáticas, y especialmente las bases de datos, dentro de la disciplina.

En un plano internacional, esta tendencia hacia la estandarización y explicitación de los criterios de registro (o cuando menos hacia su discusión) queda refrendada y consolidada tanto por diversos textos legales, como por ejemplo la conocida Convención de La Valeta (CoE, 1992), como por diversas iniciativas multilaterales tendentes a dar homogeneidad y consistencia a los criterios de registro y descripción de datos de yacimientos arqueológicos. Este es el caso de, por ejemplo, el Draft International Core Data Standard for Archaeological Sites and Monuments (CIDOC 1995; Quine y Hansen, 1996; Guillot y Hansen, 2000), de varias revisiones internacionales recientes de criterios y procedimientos de catalogación de yacimientos arqueológicos, caso de la Caere Survey (Mos-
A los colegas arqueólogos y arqueólogas, el TPHA propone ante todo un posible marco conceptual con el que concebir la integración de la información arqueológica dentro un marco más genérico, el del Patrimonio Histórico y Cultural.

cati y Tagliamonte, 1998) o la World Survey of National Archaeological Site Records realizada por el Archaeological Sites Working Group del CIDOC (CIDOC-ASWG, 2000) o de diversos tesauros y glosarios temáticos multilingues como los ya editados de yacimientos europeos de la Edad del Bronce (CoE, 1995) y Egiptología (Bergman, 1994; Van der Plas, 1996).

Entre las iniciativas que por su gestación y desarrollo no pueden ser calificadas de internacionales, aunque desde luego han ido orientadas hacia una estandarización y normalización (de ámbito supra-nacional) de los criterios de descripción, estructuración, presentación, etc. de la información arqueológica, destacan los varias "guías de buen uso" que el Archaeology Data Service de la Universidad de York ha venido publicando desde su creación (Gillings y Wise, 1998; Bewly et alii, 1998; etc.).

Similarmente, los diversos tesauros arqueológicos publicados en los últimos 20 años han tenido como uno de sus objetivos principales lograr una mayor consistencia y eficacia en la descripción y organización de documentación arqueológica. Avances significativos (aunque no específicamente arqueológicos) en esta linea de trabajo son el bien conocido tesauro de arte y arquitectura de la J. Paul Getty Trust (Petersen, 1990) y el tesauro de arquitectura del Ministerio Francés de Cultura (Chatenet y Verdier, 2000). Un caso especialmente interesante y de mucha mayor relevancia arqueológica es el de los tesauros publicados en el Reino Unido desde mediados de los 1980 por English Heritage, la Royal Commission on the Historical Monuments of England (RCHME, 1986; 1989; 1992; 1996; RCHME-EH, 1995; 1998) y la Museum Documentation Association (MDA, 1997).

Es dentro de esta tendencia disciplinar, y en el marco del objetivo general de facilitar la entrada y recuperación de la información patrimonial dentro de bases de datos informáticas, donde se inserta la iniciativa del TPHA (Muñoz Cruz, 1996), una iniciativa única en nuesrto país. Específicamente, las ventajas asociadas a tal normalización terminológica son: 
I. Proporcionar una estructura lógico-semántica de base con la que organizar una terminología que, en el caso de la Arqueología, es de creciente complejidad por la masiva introducción de aportes metodológicos y teoréticos desde otras disciplinas (desde métodos físico-químicos, hasta términos botánicos y arquitectónicos).

2. A efectos prácticos de entrada y alimentación de datos en bases de datos informáticas manejadas por múltiples operadores, evitar problemas asociados al uso subjetivo y no normalizado del lenguaje, típicamente sinonimias (solapamientos, o diferentes términos significando lo mismo) y polisemias (un mismo término con diferentes significados en función del contexto ligüistico).

3. A efectos de recuperación y consulta de datos, la normalización terminológica permite simplificar y hacer más efectiva la recuperación de datos contenidos en las bases de datos por parte de los usuarios/as.

Aparte de su función específica, desde un punto de vista más genérico, y en el marco de esa línea de trabajo que se refleja en el ámbito internacional, el TPHA puede llegar a constituirse como una herramienta de trabajo de gran utilidad para toda la comunidad hispanohablante internacional, ya que todos los tesauros previamente existentes corresponden a paises de habla inglesa y francesa.

\section{Aproximación Disciplinar vs. Aproximación Temática}

Un primer rasgo que sin duda condiciona la integración de la terminología arqueológica dentro del TPHA es el carácter temático de su estructura jerárquica, es decir, de su organización interna. En un primero momento, el Grupo de Trabajo consideró la alternativa de una aproximación disciplinar donde la terminología estuviera organizada en secciones tales como Arquitectura, Arqueología, Etnología, etc. Esta estructura discipinar habría comportado dos ventajas principales:

- En primer lugar, los usuarios se habrían encontrado más cómodos con la organización y disposición de los términos dentro de la estructura jerárquica.

- En segundo lugar, al Grupo de Trabajo le habría sido relativamente más sencillo llegar a estructuras jerárquicas parciales (disciplinares) reconocibles por (y aceptables para) una mayoría de colegas.

El grave inconveniente de esta aproximación, sin embargo, era que llevaba implícita una enorme inflación de términos, repetidos con frecuencia en diversas (o en la totalidad) de las disciplinas afectadas. Por poner solo un ejemplo, Objetos Inmuebles constituye un área de solapamiento terminológico masivo ente Arqueología y Arquitectura. Dado que la Arquitectura se ha interesado tradicionalmente por los sistemas y técnicas constructivas de todas las épocas (incluidas aquellas para las que la Arqueología constituye la úni- ca aproximación científica viable), y que desde hace algunas décadas la metodología arqueológica se ha comenzado a aplicar con éxito al análisis de paramentos (para la rehabilitación y restauración de edificios históricos), ambas disciplinas comparten un amplio abanico de intereses $y$, desde luego, una respetable cantidad de vocabulario. Si tomamos un término cualquiera al azar, por ejemplo "Revoco" ("Técnica de Revestimiento Continuo") la aproximación disciplinar hubiera requerido bien duplicarlo (o triplicarlo, puesto que posiblemente también habría sido reclamado como término etnológico) o bien entrar en la imposible discusión de si dicho término correspondería al ámbito de la práctica arquitectónica $\mathrm{O}$ al de la arqueológica.

Siguiendo con el ejemplo del solapamiento entre Arquitectura y Arqueología en el dominio de Bienes Inmuebles, la desventaja económica de la aproximación disciplinar frente a la temática queda ilustrada en la evolución de la estrategia seguida por la propia Royal Commission on the Historical Monuments de Inglaterra (institución pionera en materia de tesauros de arqueología) en la materia. En una primera etapa se compilaron dos tesauros generales independientes sobre términos arqueológicos (RCHME, 1986) y arquitectónicos (RCHME, 1989) así como un tesauro de tipos de yacimientos arqueológicos (RCHME, 1992; Beagrie y Abercromby, 1992) que pretendía contribuir a poner algo de orden en la compleja (y algo descoordinada) maraña de estructuras de datos, terminologías y bases de datos que componen los inventarios de yacimientos arqueológicos y monumentos o SMR (Sites and Monuments Records) de cada uno de los diferentes condados ingleses. Sin embargo, en una segunda etapa, esta aproximación de corte disciplinar, que separaba las terminologías arquitectónica y arqueológica, ha sido reemplazada por una aproximación temática donde los términos de ambas disciplinas son fundidos en diferentes tesauros temáticos, uno dedicado a tipos de construcciones (RCHME-EH, 1995) y otro a materiales constructivos (RCHME-EH, 1996), es decir, la misma solución que finalmente se planteó para el TPHA (aunque con la diferencia de que en este caso un solo tesauro integra las terminologías relativas a tipos de construcciones y materiales constructivos).

Los/as usuarios/as arqueólogos/as del TPHA deben por tanto tener presente que la terminología arqueológica se subsume en una estructura jerárquica general cuya racionalidad se basa ante todo en la necesidad de posibilitar una integración flexible de todas las terminologías disciplinares representadas bajo un mismo paraguas. La importancia relativa de cada descriptor de primer nivel dentro del tesauro (Figura I), por tanto, no se establece por acuerdo sobre el tamaño o peso de cada disciplina en el dominio de la gestión patrimonial, sino de acuerdo a la propia organización temática propuesta para el mismo.

Sin embargo, si en términos de economía de términos la aproximación temática era claramente ventajosa, a efectos de la dinámica interna del Grupo de 
Trabajo tuvo un costo adicional rotundo: supuso un considerable incremento de la fricción entre los intereses, actitudes y expectativas de las disciplinas representadas, dadas las dificultades de ajustar bajo una misma estructura jerárquica de carácter temático términos procedentes de disciplinas más bien diferentes. Esta fricción fue normalmente de dos tipos:

I. Por un lado la resultante del encuentro de especialidades que en un plano teórico y epistemológico han experimentado diferentes trayectorias evolutivas y que de forma correspondiente muestran en la actualidad grados de complejidad más bien dispares. Un ejemplo típico de uno de los (literalmente) cientos de escollos de esta naturaleza con los que el Grupo de Trabajo chocó a la hora de alcanzar un consenso sobre la estructura de descriptores de primer y segundo nivel adecuada para todas las disciplinas fue el de las "Prácticas y Objetos Mágico-Religiosos". Si desde el punto de vista de la Antropología o de la Arqueología, disciplinas familiarizadas con sociedades de todo grado de complejidad social y con una amplia perspectiva cronológica y temporal de la Evolución Social, la denominación era aconsejable por su neutralidad, para la Historia del Arte parecía inaceptable agrupar bajo ese mismo descriptor tanto tótems e ídolos prehistóricos como crucifijos cristianos de reciente cronología.

Como resultado del claro desfase existente entre los parámetros teóricos y epistemológicos predominantes entre diversas de las disciplinas implicadas, el logro de una estructura consensuada resultó una tarea extraordinariamente ardua, que incrementó el número de horas de discusión y debate, al tiempo que demandaba un considerable esfuerzo de imaginación y originalidad.

2. Un segundo tipo de fricción habitual en la fase de construcción de la jerarquía fue el derivado de las diferencias existentes entre disciplinas en términos de criterios y principios de clasificación. Por tomar otro ejemplo relevante desde el punto de vista arqueológico, tal fue el caso de la dificultad encontrada a la hora de definir un sistema jerárquico único para Objetos Muebles del tipo "medios de producción" o "herramientas" (finalmente denominados Utensilios), que satisficiera y respetara las tradiciones disciplinares y metodológicas (y por tanto las expectativas de los ususarios) de la Etnología y la Arqueología. Por supuesto, las premisas de partida eran más bien diferentes, ya que las clasificaciones al uso en Etnología (AAVV, 1993) suelen basarse en clases de actividades humanas (agrupándose así los utensilios en clases relativas a procesos productivos tales como utensilios de zapatería, utensilios de cantería, etc.) mientras que las arqueológicas se basan a menudo en la morfología y/o materiales de fabricación de los propios medios de producción (utensilios apuntados, utensilios de cobre, etc.) o, en todo caso, en la materia prima procesada (utensilios para procesamiento de madera, etc.)
Por otra parte, tales clasificaciones etnológicas de utensilios, basadas en el contexto productivo o en el tipo de producto resultante, presentaban en sí mismas el inconveniente de generar una potencial redundancia de términos (martillo de zapatero, martillo de cantería, etc.) reflejando quizás variaciones morfo-tecnológicas de los objetos, que en el caso del TPHA se pretendía evitar (por razones de limitación de espacio).

La solución de compromiso alcanzada fue agrupar las denominaciones de tales objetos en términos de sus funcionalidades primarias (por ejemplo utensilios contenedores, utensilios de corte, iluminación, molienda, etc.), lo que permite respetar el carácter funcional de las clasificaciones etnológicas, al tiempo que se da una mayor margen para las clasificaciones de utensilios procedentes de contextos arqueológicos cuya funcionalidad exacta no ha sido determinada. Esta solución requirió un lento proceso de definición de tales funcionalidades primarias, de manera que se minimizara en la medida de lo posible cualquier posibilidad de ambiguedad. Estas definiciones quedan incorporadas al TPHA en forma de Notas de Alcance (NA) que pueden consultarse en el Listado Alfa-Sistemático (TPHA, 1998:748-754). Así, por ejemplo, se definió todo utensilio de corte como aquel "con un borde afilado que se utiliza mediante presión y movimiento longitudinal para seccionar otro objeto", mientras que un utensilio de molienda sería aquel "con una superficie plana que se utiliza mediante movimientos rotatorios repetitivos de presión contra otra superficie plana inmóvil para triturar, machacar o pulverizar la materia prima".

\section{Figura 1 Distribución de los Principales Descriptores por Porcentaje de Términos}

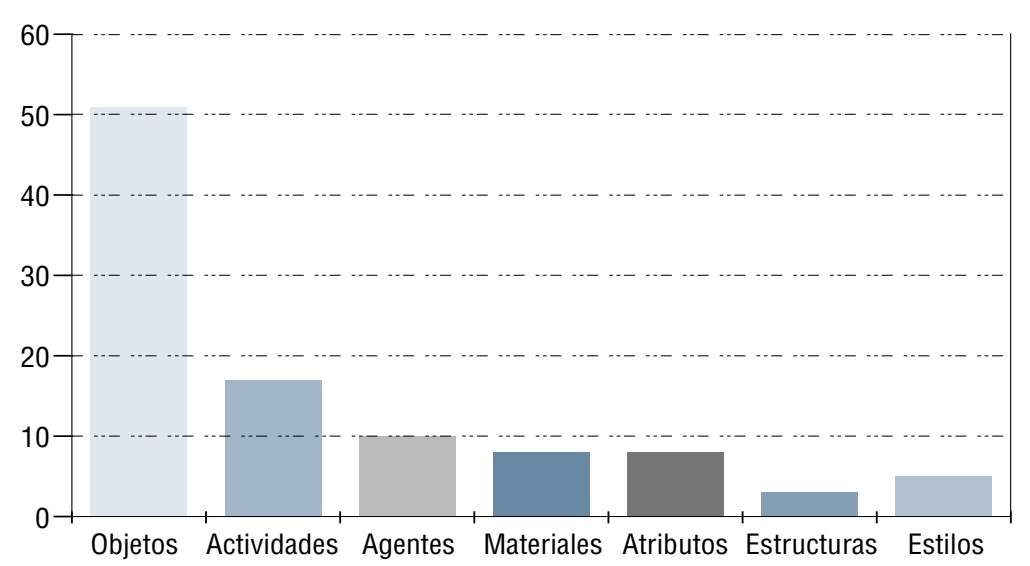

Por supuesto, la solución adoptada no carece de problemas (por ejemplo, los útiles de piedra tallada prehistóricos fueron dispuestos fuera de ese esquema general) y es probable que no satisfaga por completo a muchas/os de las/os colegas de ambos ámbitos disciplinares. Sin embargo, perfectible como sin duda es, el criterio utilizado permitió de hecho combinar eficazmente un considerable bloque de términos que en principio aparecían sometidos a clasificaciones más bien irreconciliables, posibilitando al mismo tiempo un no desdeñable ahorro de términos. 
La estructura jerárquica del TPHA resulta, por tanto, de un intenso proceso de discusión y acercamiento paulatino entre practicantes de disciplinas, no siempre tan afines como en principio cabe esperar, que reconcilia las limitaciones de espacio de la propia obra con las expectativas y tradiciones de cada una de esas disciplinas. Esta estructura alberga e integra una masa de términos que en otras experiencias (como las citadas más arriba) ha supuesto de hecho la elaboración de varios tesauros separados, resultando al propio tiempo lo suficientemente flexible como para ser capaz de acomodar la terminología arqueológica sin excesivas dificultades y proporcionar espacio para su eventual profundización.

\section{Valoración}

Quizás la forma más sincera y realista de hacer un intento de valoración del impacto y relevancia que el TPHA puede tener en la práctica arqueológica andaluza actual sea comenzar realizando un ejercicio autocrítico. En su diseño actual, y asumiendo de entrada que el TPHA 0 constituye una versión de partida de una herramienta que debe seguir perfeccionándose con el uso, es posible observar algunos de los problemas que pueden merecer una atención más especial en el futuro.

Por un lado, el laborioso proceso de crear ex novo una estructura jerárquica válida para todas las disciplinas vinculadas al Patrimonio Histórico debe ser todavía culminado. Considerando el número de términos que alberga, el descriptor de Estilos, por ejemplo, está manifiestamente sobredimensionado en la jerarquía, y podría integrarse perfectamente como descriptor segundo nivel en Estructuras. Además, Estilos contiene una serie de términos que desde el punto de vista de su utilización arqueológica necesitan ser revisados.

Pero la compilación del TPHA trajo aparejadas dificultades de otra naturaleza, aparte del complejo problema del diseño de la jerarquía, y que comentamos a continuación:

I. La profundidad del vocabulario constituye una de las limitaciones más visibles del TPHA. Esta limitación es consustancial a la necesidad de mantener dentro de unos límites operativos una masa de vocabulario que ya de por si duplica las previsiones iniciales.

2. Otras omisiones, no obstante, derivan del simple olvido de ciertos términos relevantes por parte del Grupo de Trabajo.

3. La colocación de ciertos términos dentro de la estructura jerárquica puede ser objeto de revisión. Desde el punto de vista del vocabulario arqueológico puede señalarse como ejemplo el caso de Miliarios, que aparece como Componentes de los Documentos, y que podría probablemente ser mejor emplazado como Utensilio de Señalización, junto a, por ejemplo, Mojones de Carreteras.

4. Las sinonimias, cuasi-sinonimias y polisemias fueron cuidadosamente examinadas al objeto de minimizar el impacto de estos problemas en la medida de lo posible. Para ello se compararon definiciones tomadas de los diccionarios arqueológicos más al uso (Bray y Trump, 1976; Fatás y Borrás, 1990; Ocampo, 1988; Whitehouse, 1985; Menéndez et alii, 1997; etc.) con las obtenidas en diccionarios de otras especialidades, haciéndose asimismo uso extensivo de las NA (Notas de Alcance) para matizar y precisar la acepción dada a determinados términos, así como de las UP (Usado Por) para evitar sinonimias entre términos ligeramente distintos (normalmente consolidados en disciplinas diferentes) pero con el mismo significado. Un ejemplo de una situación de este tipo es el de Vidriado Cerámico (término específico de Decoración Cerámica), bastante consolidado en la literatura y uso arqueológicos, que aparece Usado Por el término Barnizado Vítreo, más extendido en conservación (TPHA, 1998:763).

5. Finalmente, se han dado algunos errores de deletreo o escritura, fundamentalmente en el caso de denominaciones en lengua no castellana (sobre todo en latín y árabe) de agentes y objetos, que no son en absoluto infrecuentes en el TPHA dado el carácter fuertemente multicultural del Patrimonio Histórico andaluz.

La mayoría de tales problemas son en realidad fácilmente subsanables. Además, toda vez que pronto se cumplirán dos años de la salida del TPHA para uso público, en la actualidad sería de gran interés abrir una encuesta entre colegas de todos los ámbitos de la gestión del Patrimonio Histórico, para valorar qué acogida general ha tenido, y cúal ha sido la percepción por parte de sus usuarios/as de sus ventajas y problemas.

En conjunto parece posible asumir una perspectiva optimista sobre la potencial contribución de esta herramienta a la maduración de la práctica arqueológica en nuestra comunidad autónoma y en nuestro país. El TPHA es una propuesta hacia una más eficiente indización de colecciones de artefactos, edificios y lugares arqueológicos en bases de datos, así como su consulta por los usuarios, y puede contribuir a dar mayor consistencia y homogeneidad a la organización de ciertos segmentos de información arqueológica.

A los colegas arqueólogos y arqueólogas, el TPHA propone ante todo un posible marco conceptual con el que concebir la integración de la información arqueológica dentro un marco más genérico, el del Patrimonio Histórico y Cultural. 
AAVV (1993): Sistema de Clasificación de Documentación Etnográfica Española. Anales del Museo del Pueblo Español. Madrid. Ministerio de Cultura

BEAGRIE,N.-ABERCROMBY,D. (1992): "The Archaeological Syte Types Thesaurus." Thesauri for Museum Documentation. Museum Documentation Association Occasional Paper 18. Museum Documentation Association

BERGMAN, D. (1994): "EDOC: Egyptological Documentation on Computer. New Relational Database Structure, New Multiple Window User Interface and Interface to the Multilingual Thesaurus." Informatique et Égyptologie 9, 10-16.

BEWLEY, R. - DONOGHUE, D. - GAFFNEY, V. - VAN LEUSEN, M. - WISE, A. (1998): Archiving Aerial Photography and Remote Sensing Data: A Guide to Good Practice. York. Arts and Humanities Data Service, University of York. York. Arts and Humanities Data Service, University of York.

BRAY, W. - TRUMP, D. (1976): Diccionario de Arqueología. Barcelona. Labor

CHATENET, M. - VERDIER, H. (2000): Thesaurus de l'Architecture. Paris. Direction de l'Architecture et du Patrimoine

CIDOC (1995): Draft International Core Data Standard for Archaeological Sites and Monuments. Paris. CIDOC

CIDOC-ASWG (2000): Survey of National Archaeological Site Records

http://www.natmus.min.dk/cidoc/archsite/survey/survey.htm

CoE (1992): European Convention on the Protection of the Archaeological Heritage (La Valeta 16/1/1992). European Treaty Series 143. European Council. Strasbourg

CoE (1995): European Bronze Age Monuments. A Multilingual Glossary of Archaeological Terminology. Amersfoot. Council of Europe Glossary SS7

FATÁS, G. - BORRÁS, G. (1990): Diccionario de Términos de Arte y Elementos de Arqueología, Heráldica y Numismática. Madrid. Alianza

GUILLOT, D. - HANSEN, H. J. (2000): A European Core Data Standard for Archaeological. Sites and Monuments

En http://www.natmus.min.dk/cidoc/ archsite/coredata/e_cds.htm (consultado Abril 2000)

GILLINGS, M. - WISE, A. (1998): GIS Guide to Good Practice. York. Arts and Humanities Data Service, University of York.

IAPH (1998): Tesauro de Patrimonio Histórico Andaluz. Sevilla. Instituto Andaluz del Patrimonio Histórico
MDA (1997): Archaeological Objects Thesaurus. Museum Documentation Association, English Heritage y Royal Commission on the Historical Monuments of England

MENÉNDEZ, M. - JIMENO, A. - FERNÁNDEZ, V. (1997): Diccionario de Prehistoria. Madrid. Alianza Universidad

MOSCATI, P. - TAGLIAMONTE, G. (Eds.) (1998): Methodological Trends and Future Perspectives in the Application of GIS to Archaeology. Archeologia e Calcolatori 9. Firenze. All' Insegna del Giglio

MUÑOZ CRUZ, V. (1996): "La normalización del Sistema de Información del Patrimonio Histórico de Andalucía: el Tesauro de Patrimonio Histórico." Boletín del Instituto Andaluz del Patrimonio Histórico 14, 100-I I5. Sevilla. IAPH

OCAMPO, E. (1988): Diccionario de Términos Artísticos y Arqueológicos. Barcelona. Montesinos

PETERSEN, T. (Ed.) (1990): Art and Architecture Thesaurus. New York. Oxford University

QUINE, G. - HANSEN, H. (1996): "Archaeological Sites Workgroup Report." CIDOC Newsletter 7.

RCHME (1986): Thesaurus of Archaeological Terms. London. Royal Commission on the Historical Monuments of England

RCHME (1989): Revised Thesaurus of Architectural Terms. London. Royal Commission on the Historical Monuments of England

RCHME (1992): Thesaurus of Archaeological Site Types London. Royal Commission on the Historical Monuments of England

RCHME (1996): Thesaurus of Building Materials. Swindon. Royal Commission on the Historical Monuments of England

RCHME-EH (1995): Thesaurus of Monument Types. A Standard for Use in Archaeological and Architectural Records. Swindon. Royal Commission on the Historical Monuments of England and English Heritage

RCHME-EH (1998): MIDAS. A Manual and Data Standard for Monument Inventories. Swindon. Royal Commission on the Historical Monuments of England and English Heritage

VAN DER PLAS, D. (Ed.) (1996): Egyptological Thesaurus. Utrech-Paris. ICOM Comite International pour I'Egyptologie (CIPEG) and International Association of Egyptology Computer Working Group

WHITEHOUSE, R. (Ed.) (1985): MacMillan Dictionary of Archaeology. London. MacMillan Press 